\title{
Convergence Numerically of Trinomial Model in European Option Pricing
}

\author{
Entit Puspita, Fitriani Agustina, Ririn Sispiyati \\ FMIPA, Universitas Pendidikan Indonesia
}

ARTICLE INFO

A B S T R A C T

Keywords:

convergence,

option valuation

trinomial model

\begin{abstract}
A European option is a financial contract which gives its holder a right (but not an obligation) to buy or sell an underlying asset from writer at the time of expiry for a pre-determined price. The continuous European options pricing model is given by the Black-Scholes. The discrete model can be priced using the lattice models ih here we use trinomial model. We define the error simply as the difference between the trinomial approximation and the value computed by the Black-Scholes formula. An interesting characteristic about error is how to realize convergence of trinomial model option pricing to Black-Scholes option pricing. In this case we observe the convergence of Boyle trinomial model and trinomial model that built with Cox Ross Rubenstein theory.
\end{abstract}

Corresponding author:

entitpuspita@yahoo.com
(C) 2013 IRJBS, All rights reserved.

\section{INTRODUCTION}

Option is a contract between writer and holder which gives the right, not obligation for holder to buy or sell an underlying asset at or before the specified time for the specified price. The specified time called as expiration date (maturity time) and the specified price called as exercise price (strike price). Option call (put) allow holder to buy (sell) underlying asset with strike price $\mathrm{K}$. Holder can exercise European-style option just only at maturity time $\mathrm{T}$, whereas American-style option holder can exercise the option anytime during before maturity time.

Option pricing models first introduced by Black and Scholes and Merton at 1973 (Black \& Scholes,
1973). They observe behavior lognormal from stock price and then they reduce a differential partial equation which describe option pricing. For European options, they have derived a closed form of the PDP solution known as Black-Scholes formula.

Besides the research to determine the option pricing analytic solution, also developed numerical approach for option pricing. Hull and White states that the two numerical approaches are often conducted to determine the value of a derivative is by using finite difference methods and lattice methods [Hull \& White, 1990]. The lattice method consists of binomial method (binomial model), trinomial method (trinomial model), and 
multinomial method (multinomial model). In this paper we study the principles of trinomial models and, since we can treat them as an approximation of the continuous time model, their convergence.

\section{METHODS}

Binomial model in option pricing theory has a weakness, that binomial model is not too flexible because its model only looked at two possibility of stock price movement that is the stock price rises with probability $\mathrm{p}$ and the stock price down with probability $q=(1-p)$. In fact, there are many possible of stock price movements, such as trinomial model involving three possibilities stock price movement and multinomial model involving $\mathrm{n}$ possibilities stock prices movement, so these model are more flexible in bridging the real conditions in the financial markets. The structure of the stock price movement involving three possibilities can be described in the form of a tree known as the trinomial tree. In this paper, the scenario of stock price movement can seen as a part of the roots structure of the trinomial tree movement which moving towards the ends of the branches of the tree to the right of the trinomial.

As binomial models, trinomial model of $n$ period is built based on trinomial model of one period. The trinomial model of one period is stock market (trading) model with one period (one time step), in other words, in this model there are only two trading time, which is $\mathrm{t}=0$ and $\mathrm{t}=1$. As discussed earlier, at the end of period or when $t=1$, the stock price movement has three possible. First is increase with factor of increase (u) and probability $\left(p_{u}\right)$, second is steady with factor of steady $(m)$ and probability $\mathrm{p}_{\mathrm{m}}$, third is descend with factor of descend (d) and probability $\left(\mathrm{p}_{\mathrm{d}}\right)$. Suppose $\mathrm{S}_{0}$ stated the stock price at the time $t=0$, then at the end of the period $S_{0}$ could turn out to be $S_{1}\left(\omega_{1}\right), S_{2}\left(\omega_{2}\right)$, or $S_{3}\left(\omega_{3}\right)$. Later in the market with one period trinomial model is composed of two assets are risky assets, namely stocks and the risk-free asset in the form of savings deposits in banks. $B_{t}$ stated the amount of savings in the form of deposits in the bank at the time of $\mathrm{t}$ and $\mathrm{S}_{\mathrm{t}}$ stated stock price at the time $\mathrm{t}$.

In this model the movement of deposits be held deterministically, and can be expressed as follows:

$\mathrm{B}_{1}=(1+\mathrm{r})^{\mathrm{t}}$

where $r$ is the risk-less (risk-free) interest rate. Moreover need to know that on the money market applicable interest rates on bank deposits each period rate $r$ and is assumed to be applicable the following relationship:

$\mathrm{d}<1+\underline{r}<u$

equation (2) also can be expressed with:

$\mathrm{d}<\mathrm{e}^{r}<u$

Besides the foregoing assumptions, on the trinomial models there are other assumptions that is:

$\mathrm{d}<m<u$

At the end of period 1, the portfolio will become $\mathrm{V}_{1}$ which consists of $\theta_{0} \mathrm{~S}_{0}$ in shares of stock and something in the form of deposit or loan will be increase become $e^{r}\left(\mathrm{~V}_{0}-\theta_{0} \mathrm{~S}_{0}\right)=e^{r} B_{0}$ Portfolio at the end of period 1 can be expressed as follows:

$$
\begin{array}{llll} 
& { }_{0} S_{u} & e^{r} B_{0} & \mathrm{C}_{u} \\
V_{1} \quad & { }_{0} S_{m} & e^{r} B_{0} & \mathrm{C}_{m} \\
& { }_{0} S_{d} & e^{r} B_{0} & \mathrm{C}_{d}
\end{array}
$$

Based on equation (4) is known that replication portfolio in first period trinomial model is a system of linear equations consists of 3 equations and 2 variables. Therefore, a necessary and sufficient condition is that :

$m \quad d C_{u} \quad u \quad d C_{m} u \quad m C_{d} \quad 0$

According that condition, "most" contingent claims are not replicable, as a consequence the trinomial market is not complete (Takahashi, 2000). 
Takahashi have an idea to solve the problems incomplete market model in trinomial models is by assuming the embedded complete market model in the incomplete market model (Takahashi, 2000). At one period trinomial model will be obtained three embedded "complete markets" from the original incomplete market, that is $\mathrm{Q}_{(\mathrm{u}, \mathrm{m})}=\left\{\mathrm{p}_{(\mathrm{u}, \mathrm{m})}, \mathrm{q}_{(\mathrm{u}, \mathrm{m})}=1-\mathrm{p}_{(\mathrm{u}, \mathrm{m})}\right\}, \mathrm{Q}_{(\mathrm{m}, \mathrm{d})}=$ $\left\{\mathrm{p}_{(\mathrm{m}, \mathrm{d})}, \mathrm{q}_{(\mathrm{m}, \mathrm{d})}=1-\mathrm{p}_{(\mathrm{m}, \mathrm{d})}\right\}$, and $\mathrm{Q}_{(\mathrm{d}, \mathrm{u})}=\left\{\mathrm{p}_{(\mathrm{d}, \mathrm{u})}, \mathrm{q}_{(\mathrm{d}, \mathrm{u})}=\right.$ $\left.1-\mathrm{p}_{(\mathrm{d}, \mathrm{u})}\right\}$ (Takahashi, 2000). So, European option pricing trinomial model expressed in terms of the following equation:

$V_{j, i}=e^{-r n \Delta t}\left[\hat{p_{u}^{\wedge}} \cdot C_{j+1, i+1}+\hat{p_{m}} \cdot C_{j+1, i}+\hat{p_{d}} \cdot C_{j+1, i-1}\right]$

with $\mathrm{n}$ stated boundary interval and i stated stock price level.

One way to construct a trinomial models is to view this as a representation of the two-period binomial models. The construction concept can be applied to all other standard binomial models with constant volatility. Binomial models were used to represent the trinomial models are Cox Ross and Rubbenstein binomial model (Cox, Ros, \& Rubenstein, 1979). Trinomial model that built by Cox Ross and Rubenstein theory, in this study we call as CRR trinomial model. Other binomial models that can be used to construct the trinomial model is binomial model developed by Jarrow and Rudd (Jarrow \& Rudd, 1979), and binomial model developed Tian (1999). Parameters u and d on CRR trinomial models are $u=e^{\sigma \sqrt{n \Delta t}}$ and $d=e^{-\sigma} \sqrt{n \triangle t}$ with $\mathrm{m}=1$. According that, so for the parameters $\mathrm{p}_{\mathrm{u}}$ and $\mathrm{p}_{\mathrm{d}}$, obtained:

$$
p_{u}=\left(\frac{\exp \left(\frac{r \Delta t}{2}\right)-\exp \left(-\sigma \sqrt{\frac{\triangle t}{2}}\right)}{\exp \left(\sqrt[\sigma]{\frac{\triangle t}{2}}\right)-\exp \left(-\sigma \sqrt{\frac{\triangle t}{2}}\right)}\right)^{2}
$$

$$
\begin{aligned}
& p_{d}=\left(\frac{\exp \left(\sigma \sqrt{\frac{\triangle t}{2}}\right)-\exp \left(\frac{r \Delta t}{2}\right)}{\exp \left(\sigma \sqrt{\frac{\triangle t}{2}}\right)-\exp \left(-\sigma \sqrt{\frac{\triangle t}{2}}\right)}\right)^{2} \\
& p_{m}=1-p_{u}-p_{d}
\end{aligned}
$$

Other than that, there are other ways to construct trinomial models by applying that is basic assumptions and restrictions that are used in the binomial models [11]

$p_{u}+p_{m}+p_{d}=1 ; 0<p<1$

$p_{u} \cdot S \cdot u+p_{m} \cdot S \cdot m+p_{d} \cdot S \cdot d+S \cdot M$

$p_{u}\left(S^{2} u^{2}-S^{2} M^{2}\right)+p_{m}\left(S^{2} m^{2}-S^{2} M^{2}\right)$

$+p_{d}\left(S^{2} d^{2}-S^{2} M^{2}\right)=S^{2} V$

with $M=\exp (r \triangle t)$ and $V=M^{2}\left(e^{\sigma^{2}} \Delta t-1\right)$

The first trinomial models was presented by Phelim Boyle at 1986 (Boyle, 1986). Later in 19t88 Boyle extended this approach for two state variables (Boyle, 1988). Using equations (10) - (12), and setting, Boyle solved explicit expressions for transition probabilities (Boyle, 1988):

$$
\begin{aligned}
& p_{u}=\frac{\left(V+M^{2}-M\right) u-(M-1)}{(u-1)\left(u^{2}-1\right)} \\
& p_{d}=\frac{\left(V+M^{2}-M\right) u^{2}-(M-1) u^{3}}{(u-1)\left(u^{2}-1\right)} \\
& p_{m}=1-p u-p_{d}
\end{aligned}
$$

However, the parameters $\mathrm{u}, \mathrm{d}$, and $\mathrm{m}$ of the CRR models can not be used on the above parameters transition probabilities. This matter because, if the parameters $\mathrm{u}, \mathrm{d}$, and $\mathrm{m}$ of the CRR models used in trinomial Boyle model using transition probabilities (13) - (15), the basic assumption (10) is not fulfilled. Therefore, for the trinomial model with a transition probabilities (13) - (15) Boyle suggested the use of 
a dispersion parameter denoted by $\lambda$, where $\lambda>1$ for determination parameters $\mathrm{u}$ and $\mathrm{d}$, so obtained (Boyle, 1988):

$u=e^{\lambda \sigma \sqrt{\Delta t}} ; d=e^{-\lambda \sigma \sqrt{\Delta t}}$

\section{RESULTS AND DISCUSSION}

In this section we will study and illustrate the convergence of trinomial model in European option pricing. The following will be presented simulating European option pricing of CRR trinomial models and Boyle trinomial models with $\mathrm{S} 0=100, \mathrm{~K}=$ $110, \mathrm{~T}=1, \mathrm{r}=0.05, \sigma=0.3$ for $\mathrm{n}$ different.

According to the table 1 , table 2, table 3 . can be seen that European option pricing is obtained by using CRR binomial models, CRR trinomial models, Boyle trinomial models for four different values of $\mathrm{n}$ above, all can be said is approximations of European Black-Scholes option pricing. European option pricing using CRR binomial models approach the European Black-Scholes option pricing at the time of $n=400$, European option pricing using CRR trinomial models approach the European Black-Scholes option pricing at the time of $n=200$ and for Boyle trinomial models approach the European Black-Scholes option pricing at the time of $n=242$. Based on the table it can be seen that the European option pricing using trinomial models more quickly converge to European Black-Scholes option pricing compared to European option pricing using binomial models.

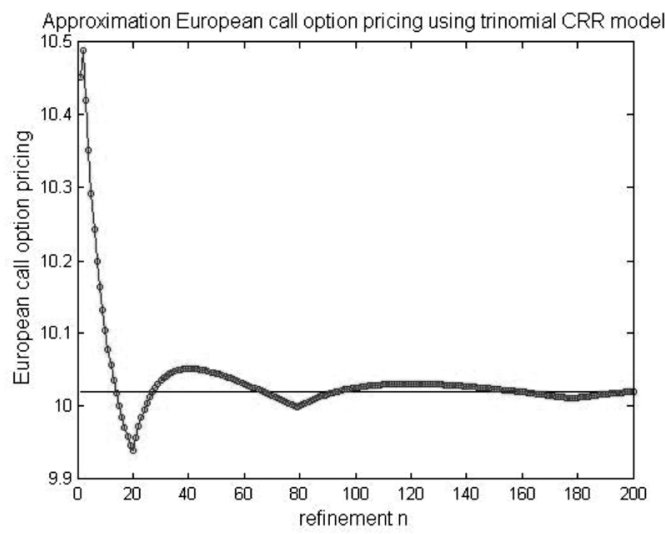

Figure 1. European call option pricing using CRR trinomial model with $S 0=100, K=110, T=1$, $r=0.05, \sigma=0.3$, for $n=1, \ldots, 200$

Table 1. Call and put European Option Pricing use CRR Binomial models

\begin{tabular}{cccccc}
\hline \multirow{2}{*}{ Type of Option } & \multicolumn{5}{c}{ European Option Pricing } \\
\cline { 2 - 5 } & $\mathbf{n}=100$ & $\mathbf{n}=200$ & $\mathbf{n}=350$ & $\mathbf{n}=400$ & Black-Scholes \\
\hline European Call & 10.0451 & 10.0257 & 10.0125 & 10.0205 & 10.0201 \\
\hline European Put & 14.6804 & 14.6609 & 14.6478 & 14.6557 & 14.6553 \\
\hline
\end{tabular}

Table 2. Call and put European Option Pricing use CRR Trinomial models

\begin{tabular}{cccccc}
\hline \multirow{2}{*}{ Type of Option } & \multicolumn{5}{c}{ European Option Pricing } \\
\cline { 2 - 5 } & $\mathbf{n}=50$ & $\mathbf{n}=100$ & $\mathbf{n}=175$ & $\mathbf{n}=200$ & \multirow{2}{*}{ Black-Scholes } \\
\hline European Call & 10.0451 & 10.0257 & 10.0125 & 10.0205 & 10.0201 \\
\hline European Put & 14.6804 & 14.6609 & 14.6478 & 14.6557 & 14.6553 \\
\hline
\end{tabular}

Table 3. Call and put European Option Pricing use Boyle Trinomial models

\begin{tabular}{|c|c|c|c|c|c|}
\hline \multirow{2}{*}{ Type of Option } & \multicolumn{4}{|c|}{ European Option Pricing } & \multirow{2}{*}{ Black-Scholes } \\
\hline & $\mathrm{n}=50$ & $\mathrm{n}=100$ & $\mathrm{n}=175$ & $\mathrm{n}=242$ & \\
\hline European Call & 10.0274 & 10.0195 & 10.0263 & 10.0202 & 10.0201 \\
\hline European Put & 14.6626 & 14.6547 & 14.6615 & 14.6554 & 14.6553 \\
\hline
\end{tabular}




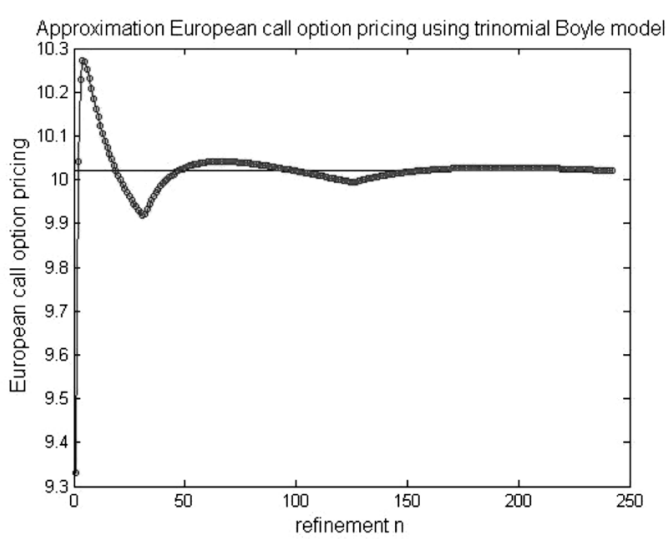

Figure 2. European call option pricing using Boyle trinomial model with $S 0=100, K=110, T=1$, $r=0.05, \sigma=0.3$, for $n=1, \ldots, 200$

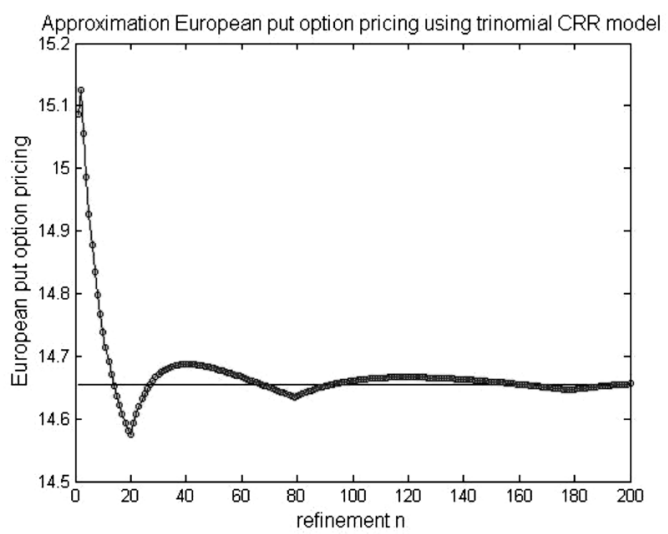

Figure 3. European put option pricing using CRR trinomial model with $S 0=100, K=110, T=1$, $r=0.05, \sigma=0.3$, for $n=1, \ldots, 200$

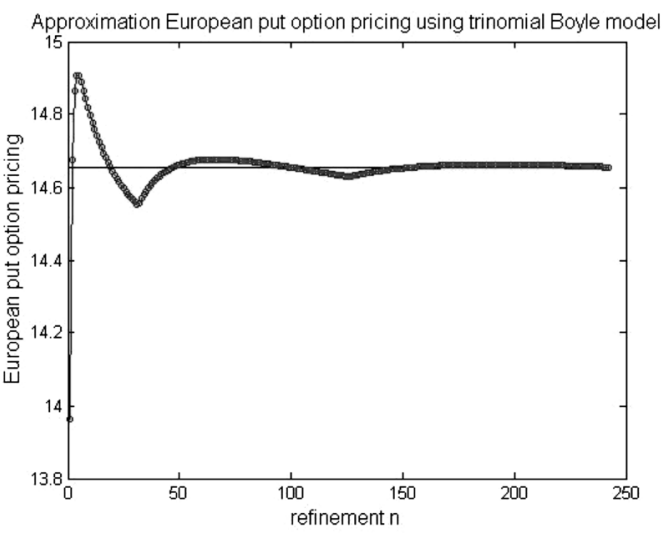

Figure 4. European put option pricing using Boyle trinomial model with $S 0=100, K=110, T=1$, $r=0.05, \sigma=0.3$, for $n=1, \ldots, 200$
Based on figure 1 - figure 4 can be known that although European option pricing CRR trinomial models and Boyle trinomial models is an approximation of European option pricing BlackScholes when $\mathrm{n}$ increasingly larger, but it turns out that convergence of European option pricing trinomial models is not monotonous, but still better than binomial models, it can be seen clearly from European option pricing trinomial models charts that moves up and down but not too volatile.

The unarragement movement of European option pricing trinomial models, resulting the option price convergence is not monotonous, but the order convergence of European option pricing trinomial models can be determined. Basically European option pricing which obtained by using trinomial models will not be the same as European Black-Scholes option pricing. Therefore, there is a difference between European option pricing trinomial models with European Black-Scholes option pricing. The difference between option pricing is referred as error. Error value of both the option price is defined as follows:

$e_{n}=\left[c\left(t_{0}, S_{0}\right)-c_{n}\left(t_{0}, S_{0}\right)\right]$

By applying the central limit theorem in equation (18) is obtained that

$\lim _{n} e_{n} 0$

This means that option pricing is determined using trinomial models will converge towards option pricing is determined using Black-Scholes formula.

Basically, it is possible to determine in what order the option pricing convergence is obtained. This may be done to determine the exact upper boundary for the equation (20). Therefore, to explain order of convergence as required under this definition (Leisen \& Reimer, 1996).

Definisi 5 (Leisen \& Reimer, 1996)

Let $f: x \longrightarrow \max \{x-K, 0\}$ be a European call 
option. A sequence of lattices converges with order $\rho>0$ if there exists a constant $\kappa>0$ such that

$e_{n} \leq \frac{\kappa}{{ }_{n} \rho^{\prime}} \forall n \in \mathrm{N}$

Furthermore, by applying the logarithm to the equation (19), be obtained

$\log \left(e_{n}\right) \leq \log \kappa-\rho \log n$

where it is shown that the logarithm error as a function of $\log n$ will be located below a straight line with a slope (slope) $-\rho$.

A lattice approach converges with order $\rho>0$ if for all $\mathrm{S}_{0}, \mathrm{~K}, \mathrm{r}, \sigma$, T the specified sequence of lattices converges with order $\rho>0$ and denote this with $e^{n}=O\left(\frac{1}{{ }_{n} \rho}\right)$. Please note that convergence of option valuation is implied by any order greater than 0. Higher order means more quickly convergence. Thus the theoretical concept of order of convergence is not unique: a lattice approach with order $\rho$ has also order $\tilde{\rho} \leq \rho$. Order of convergence is very easy to observe in actual simulations: in figures we plot the error against the refinement $n$ on a log-log-scale (Leisen \& Reimer, 1996). The following will be presented simulation error of European option pricing trinomial models with $\mathrm{S}_{0}=100, \mathrm{~K}=110, \mathrm{~T}=1, \mathrm{r}=0.05, \sigma=0.3$, for $\mathrm{n}=1, \ldots, 200$.

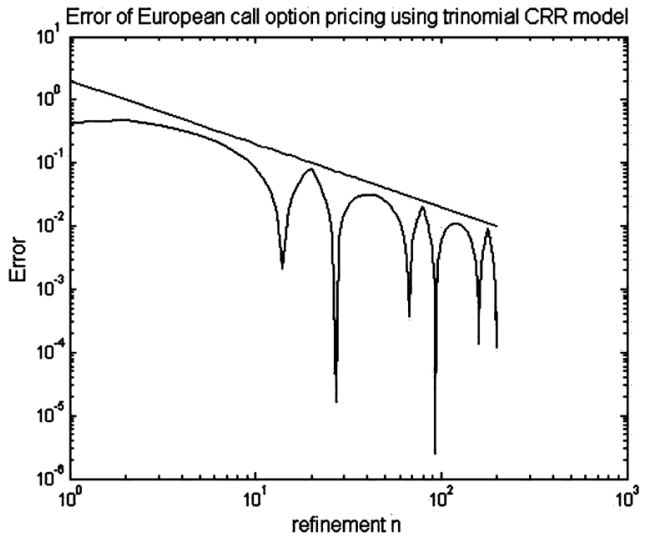

Figure 5. Error of the European call option pricing CRR trinomial models with $S 0=100, K=110, T=1, r=0.05, \sigma=0.3$,

for $n=1, \ldots, 242$ using log-log scale with $\mathrm{kappa}=3.5$, lambda $=1.5$

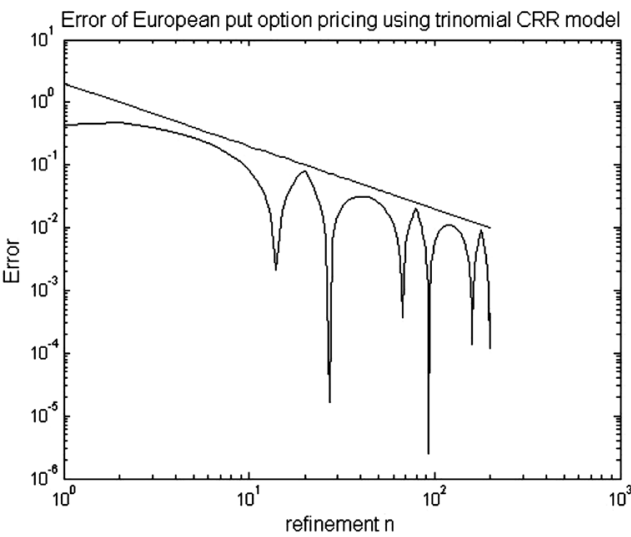

Figure 6. Error of the European put option pricing CRR trinomial models with $S O=100, K=110, T=$ $1, r=0.05, \sigma=0.3$, for $n=1, \ldots, 242$ using log-log scale with $\mathrm{kappa}=3.5$, lambda $=1.5$

Based on the simulation in the direction of European option pricing CRR trinomial models above obtained kappa value is 2 and $\rho=1.5$. Therefore order of convergence for European option pricing trinomial CRR models in this simulation is 1.5. The following will be presented simulation error of European option pricing Boyle trinomial models with $\mathrm{S}_{0}=100, \mathrm{~K}=110, \mathrm{~T}=1, \mathrm{r}$ $=0.05, \sigma=0.3$, for $\mathrm{n}=1, \ldots, 242$.

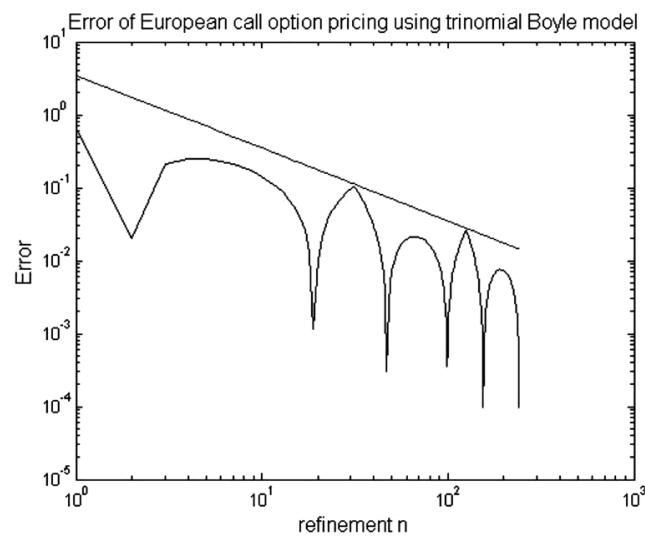

Figure 7. Error of the European call option pricing Boyle trinomial models with $S 0=100, K=110, T=$ $1, r=0.05, \sigma=0.3$, for $n=1, \ldots, 242$ using log-log scale with $\mathrm{kappa}=3.5$, lambda $=1.78$ 


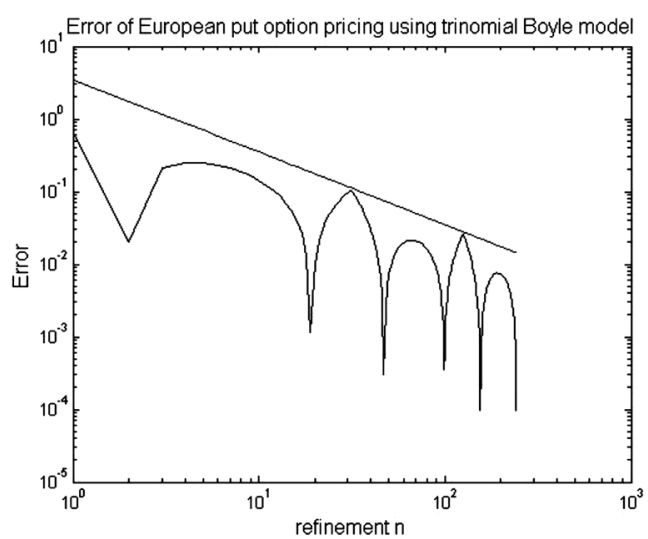

Figure 8. Error of the European put option pricing Boyle trinomial models with $S 0=100, K=110, T=$ $1, r=0.05, \sigma=0.3$, for $n=1, \ldots, 242$ using log-log scale with kappa $=3.5$, lambda $=1.78$

Based on the simulation in the direction of European option pricing Boyle trinomial models above obtained kappa value is 3.5 with lamda $=$ 1.78 and $\rho=1.85$. Therefore order of convergence for European option pricing Boyle trinomial models in this simuation is 1.85 .

\section{CONCLUSION}

Based on the overall analysis and simulations that have been done previously it can be concluded as follows:

1. European option pricing trinomial models for increasingly larger $\mathrm{n}$ will converge to Black-Scholes option pricing. Convergence of European option pricing trinomial models still not monotonous but rather steady than binomial models. European option pricing trinomial models more quickly converge to Black-Scholes option pricing than European option pricing binomial models.

2. According numerically analysis by simulation known that order of convergence for trinomial models constructed from CRR binomial models is one point five, whereas order of convergence for trinomial models proposed by Boyle to construct of the basic assumptions and restrictions general binomial models is one point eight five. Generally, order of convergence trinomial models has value more than one.

\section{REFEREN C ES}

Boyle, P. (1986). Option Valuation Using a Three-Jump Process. International Options Journal, Vol. 3, 7-12.

Boyle, P. (1988). A Lattice Framework for Option Pricing with Two State Variables. Journal of Financial and Quantitative Analysis, Vol 3, pp. 1-12.

Cox, J., Ross, S.A., Rubinstein M. (1979), Option Pricing: A Simplified Approach, Journal of Financial Economics 7, 229-263

Jarrow, R. \& Rudd, A. (1982) Approximate option valuation for arbitrary stochastic processes. Journal of Financial Economics, Vol. 10, pp. 347-369.

John Hull and Alan White, «Pricing interest-rate derivative securities», The Review of Financial Studies, Vol 3, No. 4 (1990) pp. 573-592

Leisen, Dietmar., Matthias Reimer. (1996), Binomial models for option valuation-examining and improving convergence, Applied Mathematical Finance, 3, 319-346.

Takahashi, H. (2000), A Note on Pricing Derivatives in an Incomplete Markets, Hitotsubashi University.

Tian, Y. (1999). A flexible binomial option pricing model. The Journal of Futures Markets, Vol. 19, No. 7, pp. 817-843.

Tero, Haahtela. (2010), Recombining Trinomial Tree for Real Option Valuation with Changing Volatily, Helsinki University of Technology. 